\title{
Le relazioni tra i diversi livelli di governo in Italia. Suggestioni a partire dal dibattito spagnolo sul regime locale
}

\section{The relationship between different levels of government in Italy. Inspirations from the Spanish debate on local government}

\author{
Gianluca Gardini \\ Università degli Studi di Ferrara (Italia) \\ ORCID: https://orcid.org/0000-0002-7979-723X \\ gianluca.gardini@unife.it
}

\section{NOTA BIOGRÁFICA}

Profesor de Derecho Administrativo y Derecho Público de la Información en la Universidad de Ferrara. Actualmente es Catedrático de Derecho Administrativo en la Facultad de Derecho de la Universidad de Ferrara; Profesor estable de Derecho Administrativo en la Escuela de Postgrado en Estudios de Administración Pública (S.P.I.S.A.) de la Universidad de Bolonia; Director Científico de la Revista Le Istituzioni del Federalismo; fue Defensor del Pueblo de la región de Emilia Romagna de 2013 a 2018. Fue Presidente de 2008 a 2013 del Comitato Regionale delle Comunicazioni (CORECOM) dell'Emilia Romagna.

\section{RESUMEN}

Le analogie tra Spagna e Italia investono l'intero assetto del sistema territoriale. I due paesi, dal punto di vista dell'assetto organizzativo, hanno sicuramente molti elementi in comune, soprattutto sotto il profilo del difficile equilibrio tra centro e autonomie. Le ampie convergenze tra Italia e Spagna in relazione al fenomeno territoriale non significano, tuttavia, che il bilancio debba necessariamente essere unico e condiviso. Con l'attenuarsi della crisi economica, in Italia lo Stato ha perso parte della rilevanza che aveva acquisito grazie alla gestione dell'emergenza. E' inevitabile supporre che la netta bocciatura popolare della proposta di riforma costituzionale del 2016 costringerà a ripensare l'intero sistema territoriale italiano, e a reinserire nell'agenda pubblica termini progressivamente fuoriusciti dal lessico politico, come decentramento, autonomia, federalismo.

\section{PALABRAS CLAVE}

Sistema delle autonomie locali; comparazione Italia Spagna; decentramento; federalismo; crisi economica.

\begin{abstract}
The analogies between Spain and Italy affect the hole structure of the local government. From the point of view of the organizational structure, the two countries certainly have many elements in common, especially for what concerns the difficult balance between State and local autonomies. The wide convergences between Italy and Spain in relation to the territorial phenomenon do not mean, however, that the overall assessment must necessarily be the same. As the economic crisis eased, in Italy the State has lost much of the importance it had acquired thanks to the management of the emergency. It is inevitable to suppose that the popular rejection of the constitutional reform proposal, in 2016, will force to rethink the entire Italian territorial system, and to reinsert in the public agenda terms gradually out of the political lexicon, such as decentralization, autonomy, federalism.
\end{abstract}

\section{KEYWORDS}

Local self-government system; Italy-Spain comparison; decentralization; federalism; economic crisis. 


\section{SUMARIO}

1. CONVERGENZE E DIVERGENZE TRA LE AUTONOMIE TERRITORIALI IN ITALIA E SPAGNA. 2. L'IMPATTO DELLA CRISI ECONOMICA SULLE AUTONOMIE IN ITALIA. 3. L'ERA POST CRISI E POST REFERENDUM. PRIMI SEGNALI DI CAMBIAMENTO? 4. IL TEMA SPECIFICO DELL'AREA VASTA: SPUNTI DAL DIBATTITO SPAGNOLO.

\section{CONVERGENZE E DIVERGENZE TRA LE AUTONOMIE TERRITORIALI IN ITALIA E SPAGNA}

Quelli spagnoli sono «territori inquieti», come li ha recentemente definiti Luciano Vandelli ${ }^{1}$, grande studioso del fenomeno autonomistico in Europa, il quale, nel porre in relazione l'articolazione politico-istituzionale del Regno di Spagna con il suo substrato geofisico, evidenzia il radicale cambio di prospettiva che, nell'arco di due secoli, si è verificato rispetto all'idea di territorio che la Rivoluzione francese ha reso universale.

II territorio è tradizionalmente considerato come elemento fondamentale e persino esclusivo nella rappresentanza delle comunità (una comunità, un municipio) e nell'organizzazione dello spazio politico amministrativo, ma esso ha progressivamente perso centralità nella comprensione del fenomeno sociale e dell'architettura istituzionale. Ciò, segnala Vandelli, accade non solo in Spagna, ma nell'intera area europea.

II processo di trasformazione coinvolge le relazioni tra le persone e i territori, tra le comunità e i luoghi in cui esse si radicano, rimodellando l'idea stessa di identità. Per questa ragione, secondo alcuni studiosi, l'identità avrebbe cessato di essere un elemento statico, saldato ad un territorio definito, com'era tradizionalmente nello Stato moderno, per divenire una nozione dinamica e evolutiva, sempre più condizionata dall'appartenenza a sistemi sovranazionali e globali ${ }^{2}$. In Italia il fenomeno è reso evidente dalla insoddisfazione generale rispetto a territori regionali che non corrispondono ormai più alle diverse identità culturali e sociali della popolazione ${ }^{3}$. In questo senso si è parlato di «irrazionale continuità del disegno geografico delle unità politico amministrative» italiane ${ }^{4}$, evidenziando come il disegno istituzionale delle regioni, dalle legioni militari romane alle regioni statistiche di Piero Maestri e Cesare Correnti, sia rimasto pressoché immutato nel corso dei secoli, entrando a far parte della nostra Costituzione in base a una logica statica e anacronistica ${ }^{5}$.

Non solo. Le analogie tra Spagna e Italia investono l'intero assetto del sistema territoriale e le complicate relazioni tra centro e autonomie. «Nei suoi 40 anni di vita, la Costituzione spagnola non pare avere trovato soluzione alla storica "questione territoriale" che segna la vita dello Stato spagnolo fin dalle sue origini», osservano gli studiosi della materia ${ }^{6}$. La stessa considerazione potrebbe valere per le autonomie territoriali italiane, a distanza di 70 anni dalla loro riconoscimento nella Carta costituzionale del 1948.

I due paesi, dal punto di vista dell'assetto organizzativo hanno sicuramente molti elementi in comune, soprattutto sotto il profilo del rapporto tra sovranità centralista e decentramento autonomistico. Le ampie convergenze tra Italia e Spagna in relazione al fenomeno territoriale non significano, tuttavia, che il bilancio debba necessariamente essere unico e condiviso.

Per cercare di cogliere meglio questi aspetti prenderò le mosse da un recente numero monografico della Rivista Istituzioni del Federalismo, dedicato a Quarant'anni di autonomia in Spagna, dove sono pubblicati alcuni interessanti scritti di studiosi italiani e spagnoli, sia giuristi che politologi, in cui i due sistemi vengono messi a confronto.

Il primo elemento da sottolineare, in senso differenziale, è l'approccio spagnolo rigidamente separato tra le problematiche concernenti le Regioni e i cd. nazionalismi periferici, da un lato, e quelle concernenti gli enti locali minori, dall'altro. Ciò rivela una differenza profonda nell'ambito delle due «questioni territoriali».

1 Relazione alle Jornadas sobre la organización territorial de los Estados: experiencias y problemas en América y Europa, organizzate dall'Instituto Internacional de Derecho Administrativo (IIDA), Università di Salamanca, 21 e 22 gennaio 2019.

2 PAREJO ALFONSO, L. (2019): "Las reformas territoriales en España”, in FONT I LLOVET, T.; GALÁN GALÁN, A.; TUBERTINI, C. (a cura di), Las reformas del Estado. Coloquio internacional en homenaje al prof. Luciano Vandelli. Madrid: Iustel, p. 48.

3 SESTINI, A. (1947): "Fondamenti geografici delle regioni italiane", in Ulisse, p. 307 e ss.; GAMBI, L. (1964): "L'equivoco tra compartimenti statistici e regioni costituzionali", in Questioni di Geografia, Napoli, p. 155 e ss.; ID (1990) "Regioni costituzionali e regioni altre", in Società e Storia, p. 657 e ss.; ID (1999): "Il concetto di regione non come sinonimo di unità amministrativa, ma come sistema territoriale. Riflessioni sul regionalismo di Pier Paolo D'Attorre”, in Memoria e ricerca, p. 196 e ss.

4 STERPA, A. (2019): "La differenziazione possibile: istituire le macro-Regioni per ridisegnare la mappa delle diversità", in Diritti regionali, n. 2/2019; DE GIORGI CEZZI, G.; P. L. PORTALURI, P. L. (a cura di) (2016): "La coesione politico-territoriale”, Firenze; LUCARELLI, A. (2015): "Le Macroregioni «per funzioni» nell'intreccio multilivello del nuovo tipo di Stato", in Federalismi, p. 6 e ss.

5 CASSESE, S (2018): "Le nostre regioni e i pasticci da evitare", in Corriere della sera.

6 BALDI, B. (2019): "Lo stato delle autonomie: un bilancio fra luci e ombre", in Istituzioni del Federalismo, p. 299 e ss. 
Questa netta scissione tra regionalismo e localismo non trova equivalente nell'ordinamento italiano, dove è invece presente una visione più continuista, o comunque meno divaricata, del fenomeno autonomistico. Nel nostro paese la questione autonomistica rappresenta un «blocco unitario», dal punto di vista concettuale, al quale vengono ricondotte le diverse problematiche che fanno capo ai livelli di governo comunale, provinciale e regionale.

In Italia il «sistema delle autonomie» identifica una modalità di organizzazione del potere pubblico nato per contrapposizione al metodo accentratore e uniformante inaugurato dai governi post-unitari, valorizzato dall'esperienza fascista, e riproposto periodicamente dalla legislazione dell'emergenza e della crisi. All'interno della «famiglia delle autonomie territoriali» non si registra dunque quella spaccatura netta tra enti maggiori (Regioni) ed enti minori (comuni, province, città metropolitane), che caratterizza invece il Regno di Spagna. $E$, seppure una articolazione viene fatta tra i diversi livelli di governo, non è percepita come una contrapposizione, dal punto giuridico e politico, ma piuttosto come una differenziazione.

In Spagna, per contro, viene enfatizzato «el carácter bifronte del Régimen Local, deducido por el TC de lo dispuesto en el art. 149.1.18. ${ }^{\circ} \mathrm{CE}$, que reserva al Estado la legislación de las Bases del Régimen Local, y a las Comunidades Autónomas su desarrollo, o-mejor-complemento» ${ }^{7}$.

Ancora, in Spagna il progetto autonomistico originario si caratterizza in senso fortemente dualistico, e nell'ambito di esso le «nazionalità storiche» vengono collocate su un piano differente rispetto alle altre regioni, poiché solo alle prime viene consentito accedere immediatamente al livello superiore di autonomia. La Costituzione spagnola del 1978 «delinea un quadro a geometria variabile che pure rinvia alla negoziazione, approvazione e possibile riforma degli statuti regionali. Essa riconosce un livello ordinario di autonomia a tutte le comunità che si costituiscono (art. 148), ma anche la possibilità di un livello superiore, acquisendo competenze che sarebbero altrimenti riservate allo Stato (art. 149), attraverso negoziazioni fra la singola regione e il governo/parlamento nazionale nella fase di elaborazione dello statuto di autonomia» ${ }^{8}$. Le Regioni spagnole non devono necessariamente coprire l'intero territorio dello Stato, non sono previste in numero predeterminato dalla Costituzione, dunque non sono enti costituzionalmente necessari.

Ė solo la sottoscrizione degli Accordi autonomici del 1981, siglati fra i due principali partiti statali (UCD al governo e PSOE all'opposizione), che consente di generalizzare e uniformare il processo di autonomia, approvando in blocco tutti gli statuti delle comunità ordinarie e dotandole di un'assemblea legislativa. Si innesca, a partire da questo momento, una rincorsa permanente tra regioni storiche e regioni che aspirano all'autonomia. Una dinamica che, lungi dal risolvere le tensioni tra stato e territori, determina semmai la mobilitazione dei partiti autonomisti contro quella che viene definita «la deriva "uniformadora" dello Stato Autonomico». In sostanza, e questa è la seconda grande diversità tra Spagna e Italia che si vuole qui sottolineare, il regionalismo spagnolo nasce differenziato, asimmetrico in favore di alcune regioni storiche, e progressivamente subisce una torsione verso l'uniformità e l'omogeneizzazione sotto la spinta degli altri territori che puntano a ottenere maggiore autonomia.

Questa spinta, di riflesso, provoca una nuova fuga in avanti delle «nazionalità storiche» (Catalogna in primis), le quali rifiutano l'assimilazione giuridica alle altre regioni, soprattutto dal punto di vista fiscale. Secondo i dati del 2018, il 61 per cento dei cittadini catalani auspica un rafforzamento dell'autonomia regionale, mentre cresce rapidamente in questa regione il favor per l'indipendenza, salito di oltre l'11 per cento negli ultimi 10 anni, ossia a partire dall'inizio della crisi economica ${ }^{9}$.

II regionalismo italiano, al contrario, è originariamente immaginato come uniforme nella Costituzione del 1948, fatta eccezione per tre regioni (a cui in seguito se ne aggiungeranno altre due) che da subito godono di un'autonomia speciale in ragione delle peculiarità geografiche, sociali e culturali. Le Regioni italiane sono previste come enti a numero chiuso, nominalmente indicati in Costituzione, che devono essere necessariamente presenti su tutto il territorio nazionale. Lo Stato italiano è regionale, non regionalizzabile, e ad eccezione di alcune regioni che hanno storicamente ottenuto un trattamento differenziato a causa delle loro peculiarità geografiche e sociali, il regime giuridico delle regioni è previsto nella Costituzione del 1948 come uniforme. Solo con la riforma del Titolo V della Costituzione, avvenuta nel 2001, viene introdotta nel testo costituzionale una norma di apertura al cd. regionalismo differenziato, che consente di attribuire «ulteriori

COSCULLUELA MONTANER, L. (2019): "Reflexiones para una reforma de la administración local en España”, in Istituzioni del Federalismo, p. 367 e ss.

8 BALDI, B. (2019): "Lo stato delle autonomie: un bilancio fra luci e ombre", cit.

9 GARCIA LUPATO, F. (2019): "Reforma constitucional y modelo territorial. Preferencias ciudadanas y propuestas partidistas entre el 40 aniversario de la Constitución española y las elecciones de 2019" in Istituzioni del Federalismo, p. 399 e ss. 
forme e condizioni particolari di autonomia» alle Regioni che ne facciano richiesta. Norma che, dopo 17 anni di letargo, ha conosciuto un improvviso risveglio nel 2017 grazie alle iniziative concrete assunte da alcune regioni italiane, che, a seguito della bocciatura referendaria della proposta di riforma costituzionale avvenuta nel 2016, hanno iniziato ad invocare maggiore autonomia rispetto alle altre in ragione dei propri meriti nella gestione delle competenze e delle risorse. L'insoddisfazione di alcune Regioni italiane rispetto all'efficienza dell'attuale assetto autonomistico non risparmia critiche nemmeno all'indirizzo delle Regioni a statuto speciale, la cui specialità oggi non sembra più potersi giustificare in base a fattori culturali o identitari, ma -si afferma- andrebbe mantenuta sulla base dell'utilizzo che queste Regioni hanno fatto della propria specialità, nella risoluzione dei problemi di convivenza tra gruppi diversi in determinati territori, nello sviluppo di tali territori, nella riduzione delle differenze in termini di sviluppo rispetto alla media nazionale.

Per quanto concerne «el gobierno local», i punti di contatto tra l'ordinamento spagnolo e quello italiano sembrano molto più evidenti, sia sotto il profilo del regime giuridico che delle criticità.

La dottrina spagnola, nell'indicare una possibile strategia di riforma dell'ordinamento locale, elenca una ad una, con straordinaria convergenza di vedute, le priorità fissate nell'agenda politica italiana. Per quanto riguarda la Spagna si afferma, infatti, che occorre articolare in modo più chiaro le relazioni tra il livello regionale e quello locale, attribuendo in modo pieno alle regioni (comunidades autonomas) la competenza normativa in materia di gobiernos locales ${ }^{10}$. Si tratta della cosiddetta «interiorización autonómica del régimen local», che a tutt'oggi solleva un acceso dibattito all'interno della dottrina spagnola ${ }^{11}$. Nell'ottica di un parallelismo con il nostro ordinamento, basterà ricordare le polemiche sollevate dal d.d.I. costituzionale Renzi-Boschi, che consegnava al legislatore statale l'intera disciplina dell'ordinamento locale, ivi compresa l'intelaiatura delle forme associative e degli enti di area vasta (che si riteneva ormai acquisita alla competenza esclusiva regionale), compiendo un passo nella direzione opposta a quella -auspicata da molti studiosi- di un'estensione alle Regioni ordinarie di una potestà legislativa paragonabile a quella primaria assegnata alle Regioni speciali, e confermando l'attrazione dell'intero ordinamento locale nella sfera di competenza statale.

In secondo luogo, una seria riforma locale in Spagna richiede la «ruptura de la excesiva uniformidad del régimen local, con un mayor reconocimiento de la diversidad» ${ }^{12}$. Anche su questo aspetto sono notevoli le consonanze con il nostro ordinamento. Come per molti altri paesi europei continentali, le origini del modello a cui si ispira l'ordinamento locale italiano affondano nella tradizione amministrativa francese, e risalgono alle concezioni rivoluzionarie del 1789, successivamente perfezionate (ed esportate) dall'impero napoleonico: robusto accentramento, centralità dei prefetti, corrispondenza tra comunità e municipalità (da cui la parcellizzazione comunale), uguaglianza intesa come uniformità di regole. In Italia l'opzione a favore dell'uniformità avviene con le leggi di unificazione del 1865 , che procedono ad una identica attribuzione di funzioni tra i comuni, a prescindere da dimensioni e caratteristiche di questi enti. Solo con la legge 142/1990 l'uniformità viene messa realmente in discussione: attraverso il riconoscimento della potestà statutaria, il riparto delle funzioni tra livelli diversi, il regime delle aree metropolitane, si incrina l'equivalenza tra uguaglianza e uniformità. Inizia così ad affermarsi, tra repentini slanci e brusche interruzioni, l'idea della differenziazione, che si consoliderà poi con le riforme Bassanini del 1997.

In terzo luogo, si fa rilevare in riferimento all'ordinamento spagnolo, occorre migliorare l'articolazione tra i distinti livelli di governo locale, evitando duplicazioni, sovrapposizioni, disfunzioni legate alla coesistenza di più enti sul medesimo territorio. Inutile sottolineare le convergenze con il dibattito sul mantenimento in vita della Provincia, sulla stessa funzione di area vasta, che da decenni si è sviluppato nel nostro paese.

Infine, viene sottolineata l'importanza prioritaria di riformare e migliorare «el sistema de financiación local» in assenza della quale "ninguna reforma que se emprenda será valorada positivamente» ${ }^{13}$.

A questi aspetti, che sicuramente costituiscono altrettanti punti prioritari nell'agenda del riformatore italiano, se ne aggiungono un paio più specifici, legati alle peculiarità sociali e culturali dell'ordinamento spagnolo, che, pur essendo oggetto di interesse anche nel nostro paese, non rivestono analoga importanza nel dibattito interno. Nell'elenco delle riforme da intraprendere con urgenza, infatti, Galán Galán indica la «adopción de medidas eficaces para la lucha contra el denominado éxodo rural», e il «refuerzo de la garantía de la autonomía local». Per quanto riguarda il primo aspetto, el éxodo rural, esso consiste in un processo inarrestabile di

10 GALÁN GALÁN, A. (2019): "Aniversario de la Constitución española y de la Carta europea de la autonomía local: Los gobiernos locales en España”, in Istituzioni del Federalismo, n. 2/2019, p. 329 e ss.

11 Si veda infatti la difforme opinione espressa da COSCULLUELA MONTANER, L. cit.

12 V. ancora GALÁN GALÁN, A. cit.

13 Ibidem. 
svuotamento delle campagne a favore delle grandi realtà urbane. Questo fenomeno è noto mediaticamente come «España vacía» o «España vaciada».

II secondo aspetto peculiare riguarda l'accesso diretto alla giustizia costituzionale da parte degli enti locali, riconosciuto in Spagna con la Ley orgánica 7/1999, che si è però rivelato poco funzionale come tecnica di difesa della autonomia locale, sia per le restrizioni imposte nella legittimazione ad agire degli enti interessati, sia per la complessità del procedimento dinanzi al Tribunal Constitucional.

L'elemento di maggiore divergenza tra i due ordinamenti risiede sicuramente nel bilancio complessivo del «governo locale», formulato a distanza di alcuni decenni dal suo riconoscimento costituzionale: per quanto riguarda la Spagna, "en el caso de los gobiernos locales, cabe adelantar ya que el balance general debe ser muy positivo, sin perjuicio de que continúen existiendo algunas sombras» ${ }^{14}$; per quanto riguarda I'Italia, non è realistico pensare che un' affermazione altrettanto netta e positiva, al fine di sintetizzare settant'anni di autonomia territoriale nel paese, possa essere sottoscritta dagli studiosi della materia.

\section{L'IMPATTO DELLA CRISI ECONOMICA SULLE AUTONOMIE IN ITALIA}

Dopo la crisi economica, in Italia il disegno delle autonomie locali si presenta davvero incerto. Qual è la direzione attuale delle riforme? Cosa ci attende dopo la clamorosa bocciatura da parte degli elettori di un progetto di riforma costituzionale che avrebbe trasformato completamente gli equilibri interni tra centro e autonomie, rimodellando la forma di stato italiana?

Tutte le crisi economiche, da sempre, portano con sé una tendenza al riaccentramento della filiera decisionale pubblica, dovuta in larga parte alla necessità di ricorrere a soluzioni emergenziali per affrontare la congiuntura negativa.

L'idea di fondo che accompagna questo movimento centripeto è semplice e antica al tempo stesso: si ritiene che concentrare la formazione delle politiche pubbliche in un'unica postazione centrale, prevalente rispetto ad altre sedi decentrate ed autonome, consenta di ridurre al minimo la frammentazione decisionale, garantire una migliore coesione del sistema socio-economico nazionale, esercitare un controllo più efficace sulla spesa pubblica.

Nell'ultimo decennio il legislatore statale, forte del fatto di dover fronteggiare la crisi economica in corso, ha operato in modo sempre più esplicito per riappropriarsi di materie che la riforma del Titolo $V$ del 2001 aveva formalmente devoluto alla competenza regionale. La giurisprudenza costituzionale, a sua volta, ha offerto un supporto importante a questa prassi legislativa. In questo senso la Corte costituzionale ha fornito letture sempre più estensive di materie trasversali come l'«ordinamento civile», la «tutela della concorrenza», la «tutela dell'ambiente», i «livelli essenziali delle prestazioni»; ha contribuito ad avallare un'interpretazione "onnivora» della materia "coordinamento della finanza pubblica»" ${ }^{15}$; ha giustificato continue invasioni del legislatore statale nella sfera di competenza delle autonomie territoriali in virtù della «prevalenza» della materia toccata dall'intervento legislativo.

Tutto ciò ha avuto come risultato quello di riportare allo Stato scelte che la riforma costituzionale del 2001 aveva voluto riservare ai Consigli regionali, quali legislatori di prossimità più attenti alle esigenze espresse dai territori. L'interesse nazionale, di fronte alla crisi economica, ha finito per coincidere con quello dello Stato, con buona pace dell'art. 114 Cost., che, nel disegnare un modello policentrico e paritario di livelli di governo autonomi, ipotizzava che la Nazione, o meglio la Repubblica, potesse essere vista, oltre che come una comunità di persone, anche come «una comunità di comunità» ${ }^{16}$.

Un chiaro segnale di questa tendenza al riaccentramento era rinvenibile anche nella proposta di revisione costituzionale, anch'essa in larga misura figlia della crisi economica congiunturale: il progetto di riforma veniva ad aggiungere alle materie di esclusiva competenza statale l'«ordinamento dei Comuni e Città metropolitane», insieme con le «disposizioni di principio sulle forme associative dei Comuni» e «i profili ordinamentali generali relativi agli enti di area vasta» (art. 40, comma 4 d.d.I. Renzi-Boschi). In pratica il d.d.I. costituzionale consegnava al legislatore statale l'intera disciplina dell'ordinamento locale, ivi compresa l'intelaiatura delle forme associative e degli enti di area vasta (che si riteneva ormai acquisita

14 Ibidem.

15 DE MARTIN, G. C. (2006): “Unitarietà della Repubblica e pluralismo delle istituzioni politiche: l'organizzazione delle Regioni”, in LABRIOLA, S. (a cura di): Studi per il $600^{\circ}$ della costituente. Bari: Laterza.

16 FALCON, G. (2012): “A che servono le Regioni?”, in Le Regioni, p. 767. 
alla competenza esclusiva regionale), confermando l'attrazione dell'intero ordinamento locale nella sfera di competenza statale.

Non solo. Di fronte alla crisi economica, il legislatore è intervenuto facendo intenso ricorso alla decretazione di urgenza. Tuttavia, molte misure introdotte attraverso questa fonte eccezionale vengono dichiarate incostituzionali per il fatto di essere state adottate con decreto legge, in assenza dei presupposti di necessità e urgenza previsti dalla Costituzione (Corte cost., sent. n. 220 del 2013). Secondo la Corte, le componenti essenziali dell'intelaiatura dell'ordinamento degli enti locali devono essere «per loro natura disciplinate da leggi destinate a durare nel tempo e rispondenti ad esigenze sociali ed istituzionali di lungo periodo», dal momento che attraverso questi interventi si avvia «una trasformazione radicale dell'intero sistema, su cui da tempo è aperto un ampio dibattito nelle sedi politiche e dottrinali».

Tra le riforme tentate attraverso la decretazione d'urgenza (e successivamente bocciate dalla Corte) vi è quella riguardante l'ordinamento delle Province, enti ciclicamente messi in discussione nel dibattito istituzionale italiano. Le manovre economiche della crisi (2011-12) in vario modo tentano di intervenire sulle dimensioni (puntando a robusti accorpamenti), funzioni, legittimazione delle province, ma questi tentativi sono destinati a cadere sotto la scure della Corte costituzionale.

Viene dunque imboccata la strada maestra della legge parlamentare, varata in attesa di un'imminente riforma Costituzionale, secondo il modello della legge Bassanini che anticipava sul versante amministrativo la riforma del titolo $\mathrm{V}$ Cost.. La legge Delrio si auto-qualifica infatti come transitoria («in attesa della riforma del titolo $\mathrm{V}$ della parte seconda della Costituzione e delle relative norme di attuazione»).

Era evidente da tempo la necessità di riprendere il percorso di una razionale redistribuzione delle funzioni tra le diverse istituzioni locali, applicando i principi generali del nuovo articolo 118 della Costituzione. La legge n. 56/14 avvia un processo di riallocazione delle funzioni a Costituzione invariata che, per la prima volta in modo organico, cerca di assicurare una differenziazione tra i livelli di governo di area vasta e di prossimità, e di garantire dimensioni e capacità adeguate agli enti destinatari delle funzioni.

I punti di intervento della riforma sono tre:

a) Anche per rispondere alle sollecitazioni dell'Unione europea ${ }^{17}$, il legislatore italiano rivede il livello di governo intermedio, quello d'area vasta. In quest'ottica, la legge 56/14 configura le «nuove» Province come enti di secondo grado (gli organi della provincia sono formati da consiglieri comunali e sindaci dei comuni ricompresi nella circoscrizione provinciale) dotati di funzioni sovracomunali sia proprie che delegate, e al contempo come enti di servizio e coordinamento orizzontale per i Comuni (cd. casa di comuni). A seguito di questa riforma, le Province vedono limitato notevolmente il perimetro delle funzioni fondamentali, che restano essenzialmente riferite alla pianificazione territoriale provinciale di coordinamento, a determinati aspetti della tutela e valorizzazione dell'ambiente, ai trasporti, alle strade in ambito provinciale, alla rete scolastica. E, soprattutto, vedono ridimensionati il personale e le risorse a loro disposizione ${ }^{18}$.

b) Viene disposta (con legge dello Stato, si badi) la «costituzione» di dieci Città Metropolitane (CM), che subentrano e succedono alle preesistenti province. In queste aree, il territorio metropolitano coincide con quello della provincia, e gli organi della CM sono espressi solo indirettamente dalla popolazione, essendo formati da sindaci e consiglieri comunali (come per le province), salva la possibilità delle

17 Com'è noto il Consiglio direttivo dalla Banca Centrale Europea, a partire dal 2010, ha rivolto una serie di raccomandazioni puntuali all'Italia sui temi dell'organizzazione istituzionale e del riordino territoriale, mentre il monitoring team della Banca Centrale Europea ha ripetutamente sostenuto che "accorpare le Province sarebbe l'unica vera misura di taglio dei costi della politica»

18 II personale dipendente delle 107 Province in servizio alla data del $1 .^{\circ}$ gennaio 2015 era pari a 41.205. La Legge di stabilità per il 2015 stabilisce la riduzione del $50 \%$ del personale delle 76 Province delle Regioni a Statuto Ordinario. Circa 16 mila dipendenti, attraverso un apposito portale realizzato dalla Funzione Pubblica, vengono trasferiti con la procedura di mobilità o vengono collocati in pensione. Nel dettaglio: 2.564 posti in pensione; 5.505 personale dei Centri per l'impiego; 720 ricollocati presso Ministeri o tribunali; 7.185 ricollocati direttamente dalle Regioni.

La spesa corrente delle Province passa da 7 miliardi 617 milioni del 2013 a 4 miliardi 309 milioni del 2018 (-43\%). La spesa per investimenti delle Province passa da 2 miliardi 732 del 2013 a 779 milioni del 2018 (-71\%). A causa di questi tagli 13 Province (La Spezia, Verbania, Novara, Asti, Alessandria, Imperia, Terni, Ascoli Piceno, Chieti, Potenza, Varese, Salerno, Biella) finiscono in predissesto. La Corte dei Conti analizzando lo stato dei bilanci delle Province nella relazione alla Commissione per il federalismo fiscale nel 2016 li definisce «manifestamente irragionevoli» e lancia l'allarme: tagli di questa portata non possono che incidere sull'erogazione dei servizi ai cittadini ed in particolare sulla gestione, manutenzione e messa in sicurezza dei 130 mila chilometri di strade, delle oltre 7.000 scuole superiori e azzerare gli interventi per la difesa del suolo e il contrasto al dissesto. Nel 2018 inizia la ripresa degli investimenti, che salgono del $6 \%$ rispetto all'anno precedente, e nel 2019 il segnale si fa sempre più deciso, tanto che il risultato della variazione del primo semestre è di $+20,5 \%$. 
singole CM di optare, statutariamente, per l'elezione diretta del sindaco e del consiglio metropolitano. Le CM hanno una missione volta allo sviluppo strategico del territorio, alla governance di servizi, infrastrutture e reti, alle connessioni esterne, particolarmente, a livello europeo. Funzioni che si aggiungono a quelle riconosciute alle Province, e sono rivolte in via primaria alla pianificazione strategica, alla pianificazione territoriale, e complessivamente, allo sviluppo economico e sociale del territorio.

c) Si punta sulle forme di associazione e cooperazione intercomunale al fine di garantire l'adeguatezza dell'esercizio delle funzioni comunali. L'eccessiva frammentazione del tessuto comunale, fatto di enti troppo piccoli per svolgere adeguatamente persino le funzioni fondamentali, rappresenta da sempre un ostacolo alla realizzazione del principio di sussidiarietà verticale. Sin dagli anni Novanta si è cercato di puntare sulle forme associative, ora imponendole per legge, ora puntando sulla libera iniziativa comunale attraverso incentivi economici e fiscali, ma i risultati sono sempre stati abbastanza deludenti, al punto che fino a qualche anno fa il numero dei comuni ha continuato a aumentare. Per questo la legge Delrio prova a rilanciare la cooperazione tra enti, nel tentativo di persuadere i comuni a mettere in comune funzioni e servizi di prossimità, senza rinunciare alla propria identità politica e istituzionale ${ }^{19}$.

Alla riforma Delrio avrebbe dovuto seguire, nel disegno del governo dell'epoca, una profonda revisione della costituzione italiana, la più importante a partire dalla sua approvazione nel 1948 (47 art. su 139). La riforma, come noto, è stata bocciata dagli elettori nel dicembre 2016, per ragioni forse più politiche che di merito. Viene così definitivamente archiviato un ambizioso disegno di carattere sistematico, che puntava a realizzare «una Repubblica delle autonomie fondata su due soli livelli territoriali di diretta rappresentanza delle rispettive comunità: le Regioni e i Comuni». Con esso, tramonta la possibilità di rivedere l'insoddisfacente riparto di competenze legislative tra Stato e Regioni, e di costruire il Senato come sede di espressione delle istanze autonomistiche territoriali.

\section{L'ERA POST CRISI E POST REFERENDUM. PRIMI SEGNALI DI CAMBIAMENTO?}

Con l'attenuarsi della crisi economica, lo Stato perde buona parte della centralità che aveva acquisito grazie alla gestione dell'emergenza. Inoltre, è inevitabile supporre che la netta bocciatura popolare della proposta di riforma costituzionale costringerà a ripensare l'intero sistema territoriale, a rivedere gli equilibri istituzionali sin qui indicati dalla Corte costituzionale, e a reinserire nell'agenda pubblica termini progressivamente fuoriusciti dal lessico politico, come decentramento, autonomia, federalismo.

I segnali di una crescente insoddisfazione, o comunque di una maggiore diffidenza nei confronti delle politiche «neocentraliste» dei Governi della crisi, erano visibili anche prima della pronuncia referendaria. Basti pensare al repentino revirement della Corte costituzionale, che, a ridosso del voto referendario, decide improvvisamente di bloccare l'iter delle riforme concernenti la pubblica amministrazione (cd. riforma Madia), dichiarando lesive della competenza legislativa regionale residuale, nonché del principio di leale collaborazione(da ottenersi attraverso un'intesa, non meglio definita, in Conferenza Stato-Regioni), le norme della legge delega n. $124 / 15^{20}$.

In questo quadro il referendum costituzionale, che ha seccamente bocciato (tra l'altro) l'espressa attribuzione dell'ordinamento comunale al legislatore statale, potrebbe ridare fiato alla tesi di coloro che, da anni, auspicano un'estensione alle Regioni ordinarie di una potestà legislativa in materia di Enti locali analoga a quella (primaria) assegnata dalla Costituzione alle Regioni speciali. Una strategia di questo genere potrebbe essere intrapresa anche a Costituzione invariata, valorizzando la competenza legislativa residuale delle Regioni (ordinarie) in tutti gli ambiti dell'ordinamento locale non espressamente coperti dalla potestà statale esclusiva ex art. 117, comma 2, lett. p) Cost.

Segnali in questa direzione possono leggersi nelle recenti iniziative di alcune regioni (come Emilia Romagna, Lombardia e Veneto) volte ad ottenere una autonomia differenziata; che non possono essere derubricate a mere iniziative di contrapposizione politica con il governo centrale dell'epoca, ma costituiscono, appunto, una reazione da parte delle regioni al riaccentramento subito dallo Stato persona, nel tentativo di riappropriarsi del proprio ruolo di enti di legislazione e programmazione ${ }^{21}$.

19 Le Unioni di Comuni hanno raggiunto - da qualche tempo-il numero di 536; i Comuni coinvolti sono più di 3.000 , quasi il $40 \%$ del totale.

20 Cfr. sentenza n. 251/2016 della Corte costituzionale.

21 Così MORELLI, A. (2017): "Ascese e declini del regionalismo italiano. Quali prospettive dopo i referendum di Lombardia e Veneto?", in Le Regioni, p. 321 e ss. 
Se qualche differenziazione si è imposta nel governo dell'area vasta, i Comuni sono ancora sottoposti a un regime uniforme, indifferenziato, e risultano per lo più inidonei ad assumere le funzioni di prossimità. I piccoli comuni, quelli sotto i 5 mila abitanti, che rappresentano oltre il $70 \%$ dei comuni italiani, non sono adeguati ad esercitare molte funzioni di prossimità, che vengono sistematicamente attratte in sussidiarietà (ascendente) da province e Regioni.

A causa della frammentazione del tessuto comunale, oltre che per l'incapacità delle regioni di adempiere al proprio ruolo di indirizzo e di centro propulsore delle autonomie locali, sono rimasti sostanzialmente irrealizzati i tre principali obiettivi posti a base della riforma del 2001, così sintetizzabili:

1. rompere l'uniformità amministrativa, attraverso i principi di sussidiarietà, differenziazione e adeguatezza;

2. attenuare il profilo della regione come ente amministrativo, valorizzandone invece il suo ruolo di ente di legislazione e programmazione;

3. ed infine, ridurre ulteriormente l'impatto e la consistenza delle funzioni amministrative assegnate allo Stato.

Anche su questo versante, tuttavia, vi sono cambiamenti meritano qualche attenzione. A partire dal numero complessivo dei Comuni, che oggi corrisponde a 7.983. Certo, si tratta di un numero tuttora elevato; ma per la prima volta dal dopoguerra comincia a diminuire, dopo decenni di costante crescita. Negli ultimi anni, infatti, le fusioni tra comuni hanno fatto un balzo in avanti: dal 2014 ad oggi se ne contano 117, mentre dal 1990 al 2013 erano state in tutto 11. La diminuzione dei comuni registrata in Italia è assai distante dai processi realizzati, in anni passati, in un'ampia area europea (dalla Germania alla Danimarca, dal Regno Unito al Belgio, sino alla Grecia) ${ }^{22}$; ma è comunque una inversione di tendenza che non va trascurata.

Alla ricerca dell'adeguatezza dell'esercizio delle funzioni comunali, come si è visto, il legislatore del 2014 ha puntato sulle forme di associazione e cooperazione intercomunale, a partire dalle Unioni di Comuni. Anche su questo piano, può essere utile fare riferimento a qualche dato. Le Unioni di Comuni hanno raggiunto -da qualche tempo- il numero di 536; i Comuni coinvolti sono più di 3.000 , quasi il $40 \%$ del totale ${ }^{23}$. Neppure in questo caso, dunque, si tratta di risultati sconvolgenti; e i processi di associazionismo intercomunale stentano tuttora ad affermarsi in varie parti del Paese.

Per quanto riguarda le Regioni, l'anno appena concluso ha visto una forte ripresa di interesse (un ritorno di fiamma, verrebbe da dire) per il cd. regionalismo differenziato, che consente di attribuire «ulteriori forme e condizioni particolari di autonomia» alle Regioni che ne facciano richiesta. Dopo 16 anni di letargo, l'art. 116.3 ha conosciuto un improvviso risveglio grazie ad alcune iniziative concrete assunte da Emilia-Romagna, Lombardia, Veneto, che il 28 febbraio 2018 hanno portato alla sottoscrizione di «Accordi preliminari» con il Governo Gentiloni; e attraverso altre iniziative in via di assunzione da parte di molte altre Regioni. II procedimento che porta all'autonomia differenziata è regolato da scarne indicazioni contenute nell'art. 116.3, vale a dire: a) l'iniziativa è adottata dalla Regione interessata, sentiti gli enti locali; b) la Regione stessa consegue un'intesa con lo Stato; c) sulla base dell'intesa viene approvata la legge, approvata dalle Camere a maggioranza assoluta dei componenti.

Alle richieste avanzate dalle Regioni si può attribuire una valenza meramente tecnica, per garantire maggiori livelli di efficienza nelle funzioni e una razionalizzazione nel riparto di competenze con lo Stato; anche se prevale la tendenza a considerare l'attivazione del processo di differenziazione come un segnale di una nuova "fase costituente», nella prospettiva complessiva di un rilancio del regionalismo. Le Regioni, evidentemente, rivendicano quel ruolo politico e programmatorio per cui erano state ideate dai Costituenti e che sinora, anche per propria responsabilità, non sono riuscite ad assumere.

L'opportunità offerta dall'art. 116 Cost. sta nel fatto che riporta l'attenzione sulla funzione legislativa regionale, e costringe ad abbandonare la prospettiva delle regioni di enti di grande amministrazione. Non è poca cosa, considerando che con la riforma delle province molte regioni hanno accentuato la vocazione di enti di macro-amministrazione.

Tuttavia, il governo continua a mantenere una posizione fortemente autoreferenziale rispetto alla richiesta delle Regioni di attribuire in materia di governance del sistema locale. Negli schemi di intesa predisposti

22 Così, ad esempio, nella Germania Federale, prima dell'unificazione, il numero dei Comuni è passato da circa 25.000 a 8.500 ; in Belgio da 2.500 a 598; in Danimarca da 1.388 a 270 . Da ultimo, un drastico e rapido processo di accorpamenti è stato realizzato in Grecia, passando da 1.033 Comuni a 325.

23 Sull'esperienza -e sulle diverse prospettive- delle forme associative, v., con una ampia documentazione, l'Indagine conoscitiva sulla gestione associata delle funzioni e dei servizi comunali, svolta dalla I Commissione della Camera dei Deputati, 28 novembre 2016. 
dal Dipartimento affari regionali le proposte delle Regioni in materia non sono state accolte. Ciò sulla base di una valutazione negativa da parte del Ministero dell'interno, espressa peraltro per iscritto, in assenza di qualsiasi interlocuzione con la delegazione trattante.

L'unica apertura in questo ambito è quella fornita nell'art. 2 dello schema del maggio 2019, secondo cui «Alla Regione Emilia-Romagna è altresì riconosciuta una competenza complementare in ordine all'organizzazione ed all'esercizio delle funzioni amministrative locali riferite alle materie oggetto della presente intesa». Si tratta di una previsione che, per la sua genericità, rischia di produrre conflitti di competenza e comunque appare riduttiva rispetto alle ben più articolate richieste che erano state presentate.

Ciò corrisponde, del resto, ad un indirizzo generale del Governo che sembra voler escludere o comunque ridurre fortemente l'intervento delle Regioni in materia di ordinamento locale, come sembra emergere anche dalla bozza di Linee guida in materia di riforma del Testo Unico degli enti locali proposte dal Governo al Tavolo appositamente costituito presso la Conferenza Stato-Città. Le due riforme (attuazione dell'art. 116 e revisione dell'ordinamento locale) sono, del resto, inevitabilmente intrecciate, non fosse altro perché le nuove funzioni e competenze eventualmente conferite dovranno essere esercitate in attuazione del principio di sussidiarietà.

Il programma del nuovo Governo Conte-bis, all'interno di una lista di 29 punti prioritari, annuncia l'intenzione del nuovo esecutivo di «completare il processo di autonomia differenziata giusta e cooperativa, che salvaguardi il principio di coesione nazionale e di solidarietà, la tutela dell'unità giuridica e economica; definisca i livelli essenziali delle prestazioni concernenti i diritti civili e sociali, i fabbisogni standard; attui compiutamente l'articolo 119, quinto comma, della Costituzione, che prevede l'istituzione di un fondo di perequazione volto a garantire a tutti i cittadini la medesima qualità dei servizi». Nel medesimo Programma di governo si legge che «(...) È inoltre necessario rivedere il testo unico per gli enti locali, introducendo un'Agenda urbana per lo sviluppo sostenibile delle città, delle città metropolitane, di Roma capitale, attuando la legge per la valorizzazione dei piccoli comuni, sopprimendo gli enti inutili. Occorre, infine, garantire il rispetto delle autonomie a statuto speciale e la tutela delle minoranze linguistiche».

Sarà quindi opportuno monitorare con attenzione l'attuazione di questo programma politico, soprattutto in relazione allo snodo fondamentale dei rapporti Regioni-enti locali.

\section{IL TEMA SPECIFICO DELL'AREA VASTA: SPUNTI DAL DIBATTITO SPAGNOLO}

Vi è però un elemento che rappresenta qualcosa di più di un semplice punto di convergenza tra il sistema italiano e quello spagnolo: il regime giuridico della Provincia. Ė infatti verosimile che il legislatore italiano del 2014, nel ridisegnare i contorni questo ente intermedio, abbia utilizzato come modello di riferimento diretto la Diputación provincial spagnola.

In quest'ottica, la Legge 56/2014 configura le «nuove» Province come enti di secondo grado (gli organi della provincia sono formati da consiglieri comunali e sindaci dei comuni ricompresi nella circoscrizione provinciale) dotati di funzioni sovracomunali sia proprie che delegate, e al contempo come enti di servizio e coordinamento orizzontale per i Comuni (cd. casa di comuni).

Le tendenze attualmente in atto nell'ordinamento spagnolo mostrano come la Ley de modernizaciòn del 2013 abbia puntato sulle Diputaciones Provinciales quali enti di coordinamento, collaborazione e assistenza nei confronti dei piccoli municipi, generando un effetto complessivo di «provincializzazione» della vita locale $^{24}$. La medesima legge stabilisce inoltre che dev'essere la provincia, laddove i comuni con popolazione inferiore ai 20 mila abitanti non siano in grado di garantire l'esercizio delle funzioni di base, ad assumere su di sé alcuni compiti municipali (riscossione delle imposte, amministrazione elettronica e contrattazione centralizzata, prevenzione ed estinzione degli incendi), compensando in questo modo le inefficienze dei piccoli comuni. In altre parole, nell'ordinamento spagnolo la riforma del 2013, legata evidentemente alla razionalizzazione delle funzioni amministrative nel periodo di crisi, ha riportato alla ribalta il tema delle Diputaciones Provinciales, enti che fino a poco tempo fa sembravano destinati ad un ruolo marginale o addirittura alla soppressione, e che ora conoscono un nuovo momento di protagonismo.

In riferimento a questo nuovo orientamento manifestato dal legislatore spagnolo, si pone un problema molto delicato, che interessa da vicino il dibattito che si è sviluppato in Italia sulle province in seguito alla

24 COSCULLUELA MONTANER, L. (2019): "Reflexiones para una reforma de la administración local en España”, in Istituzioni del Federalismo, p. 327 e ss. 
bocciatura referendaria della riforma costituzionale del 2016: il collegamento tra funzioni e elezione diretta degli organi di governo della provincia. Anche in Spagna esiste un'accesa polemica sulle competenze da attribuire alla provincia e, conseguentemente, sulle modalità di elezione degli organi di governo provinciali. La dottrina spagnola si domanda infatti se, seguito dell'attribuzione di nuove funzioni, la provincia debba continuare a considerarsi un ente dotato di competenze solo formali e strumentali, dal momento che le funzioni provinciali risultano sostanzialmente rivolte ai comuni (provincia come «municipio di municipi», che presta attività di cooperazione e assistenza ai municipi), ovvero debbano riconoscersi alla Provincia finalità sostanziali e generali, in quanto ente posto al servizio diretto della collettività (provincia come ente che presta simultaneamente anche servizi pubblici a favore dei cittadini). La scelta in favore dell'una o dell'altra opzione, determina il tipo di legittimazione democratica da ricondurre all'ente provinciale: legittimazione indiretta o di secondo grado, nel caso in cui si opti per la natura di ente a servizio dei comuni, legittimazione diretta se invece si vuole configurare la provincia come ente prestatore di servizi, che rivolge la sua attività direttamente alla collettività ${ }^{25}$.

Del resto solo una minoranza (9) dei 28 paesi dell'Unione Europea non ha un secondo livello di autonomia locale. Si tratta di Paesi di piccole dimensioni (Cipro, Lussemburgo, Malta) o che per motivi storici hanno solo il livello comunale di base (Austria, Bulgaria, Danimarca, Lettonia, Portogallo, Slovenia). In 19 Stati su 28 esiste un secondo livello di governo locale di carattere politico e in 17 di questi 19 Paesi, questo ente è dotato di funzioni amministrative proprie, diverse da quelle comunali, e di organi di governo direttamente elettivi. Si tratta di un blocco di funzioni «core», caratteristiche degli «enti intermedi» che si concentra su ambiente (pianificazione, tutela, gestione dei rifiuti e delle acque), sviluppo economico (sostegno alle imprese e politiche per l'occupazione), trasporti (viabilità, mobilità, infrastrutture), scuola (compresa l'edilizia scolastica). In alcuni casi, a queste funzioni si aggiungono anche quelle in campo sanitario (Francia e Germania). Queste funzioni sono legate a tributi propri: c'è autonomia fiscale e agli enti di governo intermedio in Europa sono assegnate entrate tributarie, a prescindere dal sistema elettorale e di governance individuato. La situazione peculiare dell'Italia è stata segnalata anche dal Consiglio d'Europa che, in una raccomandazione del 18 ottobre 2017, ha sottolineato il livello di debolezza istituzionale e finanziario delle Province italiane, anche al confronto con gli enti intermedi degli altri Paesi Europei, che si caratterizzano invece quali istituzioni con un ruolo chiaro, funzioni chiave e risorse adeguate a svolgerle.

Mi pare molto interessante seguire l'impostazione sostanziale su cui si concentra il dibattito spagnolo, perché, come si è detto, la Spagna ha ispirato la riforma delle province introdotta in Italia con la legge $56 / 2014$. II naufragio della riforma costituzionale, di cui la riforma Delrio voleva rappresentare una sorta di anticipazione amministrativa, ha determinato la riapertura del dibattito sulle province a tutti i livelli ${ }^{26}$. Com'è noto, sono state recentemente presentate le «Linee guida in materia di riforma dell'ordinamento delle province e delle città metropolitane» ${ }^{27}$, che mirano a riportare gli enti provinciali alla precedente modalità di investitura, ossia all'elezione diretta degli organi provinciali da parte dei cittadini.

In quest'ottica, il dibattito attualmente in corso in Spagna è particolarmente interessante e utile al fine di orientare le politiche in materia di area vasta. A mio avviso è essenziale, in una corretta prospettiva di riforma, non perdere di vista il rapporto tra la tipologia di funzioni attribuite ad un ente e la sua legittimazione demo-

25 In questo senso GALÁN GALÁN, A. (2019): "Aniversario de la Constitución española y de la Carta europea de la autonomía local: Los gobiernos locales en España", in Istituzioni del Federalismo, n. 2/2019, p. 329 e ss.

26 Di recente la Corte costituzionale ha dichiarato l'incostituzionalità di alcune leggi regionali della Toscana nella parte in cui attribuiscono alla Regione le competenze già esercitate dalle Province in materia di controllo periodico su tutte le attività di gestione, di intermediazione e di commercio dei rifiuti e accertamento delle relative violazioni, e di verifica e controllo dei requisiti previsti per l'applicazione delle procedure semplificate. Nel pensiero della Consulta «Mentre, infatti, con riferimento ad altre competenze amministrative in materia ambientale, il decreto del Presidente del Consiglio dei ministri, conseguente all'accordo in Conferenza unificata, ha lasciato spazio all'attività legislativa ed amministrativa regionale, altrettanto non può dirsi per le funzioni contemplate dalle norme impugnate, che il cod. ambiente riserva espressamente alle amministrazioni provinciali. Le norme contenute nel cod. ambiente, infatti, per espressa previsione dell'art. 3-bis "possono essere derogate, modificate o abrogate solo per dichiarazione espressa da successive leggi della Repubblica"; e la mancanza di tale dichiarazione espressa nella legge n. 56 del 2014 non può essere colmata né dagli interventi legislativi regionali, né dalle intese intervenute nella Conferenza unificata». In sostanza, afferma la Corte, la trasversalità della materia «tutela dell'ambiente» e l'inevitabile intreccio che essa comporta tra competenze legislative statali e regionali non permette quindi alle Regioni di derogare a quelle norme statali, anche di carattere organizzativo, che siano riconducibili all'esigenza di assicurare standard di tutela ambientale uniformi su tutto il territorio nazionale. Cfr. Corte cost., 28 maggio 2019, n. 129.

27 V. Bozza di linee guida presentate dalla Presidenza del Consiglio dei Ministri, Conferenza Stato-città ed autonomie locali, Tavolo tecnico-politico per la "redazione di linee guida finalizzate all'avvio di un percorso di revisione organica della disciplina in materia di ordinamento delle Province e delle Città metropolitane, al superamento dell'obbligo di gestione associata delle funzioni e alla semplificazione degli oneri amministrativi e contabili a carico dei Comuni, soprattutto di piccole dimensioni". 
cratica. Questo perché, al di là della compatibilità tra il metodo dell'elezione indiretta ed il principio di autonomia $^{28}$, è la sostanza dei compiti, la concretezza delle funzioni esercitate da un ente a richiedere una certa forma di legittimazione agli occhi dei cittadini. I quali risulterebbero disorientati di fronte alla realizzazione di un modello istituzionale astratto di area vasta, forse conforme al principio di legalità e al metodo della rappresentanza democratica, ma assai poco rispondente al comune sentire e alla vita concreta di una comunità.

\section{REFERENCIAS BIBLIOGRÁFICAS}

BALDI, B. (2019): "Lo stato delle autonomie: un bilancio fra luci e ombre", in Istituzioni del Federalismo, n. 2, p. 299 e ss. URL: http://www.regione.emilia-romagna.it/affari_ist/rivista_2_2019/Baldi.pdf.

CASSESE, S. (2018): "Le nostre regioni e i pasticci da evitare", in Corriere della sera. URL: https://www.corriere.it/ opinioni/18_dicembre_28/nostre-regioni-pasticci-evitare-3ff75874-0ad9-11e9-807b-d85edec6e72a.shtml?refresh_ $c e-c p$.

COSCULLUELA MONTANER, L. (2019): "Reflexiones para una reforma de la administración local en España", in Istituzioni del Federalismo, n. 2, p. 367 e ss. URL: http://www.regione.emilia-romagna.it/affari_ist/rivista_2_2019/ Montaner.pdf.

DE GIORGI CEZZI, G.; PORTALURI, P. L. (a cura di) (2016): La coesione politico-territoriale. Firenze: Firenze University Press.

DE MARTIN, G. C. (2006): "Unitarietà della Repubblica e pluralismo delle istituzioni politiche: l'organizzazione delle Regioni", in LABRIOLA, S. (a cura di): Studi per il $60^{\circ}$ della Costituente. Bari: Laterza.

SESTINI, A. (1947): "Fondamenti geografici delle regioni italiane", in Ulisse, fasc. 3, p. 307 e ss.

FALCON, G. (2012) "A che servono le Regioni?", in Le Regioni, n. 4, p. 767. URL: http://www.rivisteweb.it/ doi/10.1443/73183. DOI: https://doi.org/10.1443/73183.

GALÁN GALÁN, A. (2019): "Aniversario de la Constitución española y de la Carta europea de la autonomía local: Los gobiernos locales en España", in Istituzioni del Federalismo, núm. 2, p. 329 e ss. URL: http://www.regione.emiliaromagna.it/affari_ist/rivista_2_2019/Galan.pdf.

GAMBI, L. (1964): "L'equivoco tra compartimenti statistici e regioni costituzionali", in GAMBI, L.: Questioni di Geografia, p. 155 e ss. Napoli: Edizioni Scientifiche Italiane.

GAMBI, L. (1990) "Regioni costituzionali e regioni altre", in Società e Storia, vol. 13, n. 49, p. 657 e ss.

GAMBI, L. (1999): "Il concetto di regione non come sinonimo di unità amministrativa, ma come sistema territoriale. Riflessioni sul regionalismo di Pier Paolo D'Attorre", in Memoria e ricerca, vol. 7, n. 3, p. 196 e ss.

GARCIA LUPATO, F. (2019): "Reforma constitucional y modelo territorial. Preferencias ciudadanas y propuestas partidistas entre el 40 aniversario de la Constitución española y las elecciones de 2019" in Istituzioni del Federalismo, núm. 2, p. 399 e ss. URL: http://www.regione.emilia-romagna.it/affari_ist/rivista_2_2019/Lupato.pdf.

LUCARELLI, A. (2015) “Le Macroregioni «per funzioni» nell'intreccio multilivello del nuovo tipo di Stato", in Federalismi, n. 6, p. 6 e ss. URL: https://www.federalismi.it/App/OpenFilePDF.cfm?artid=29074\&dpath=document\&dfi $l e=24032015125606$. pdf\&content=Le\%2BMacroregioni\%2B\%27per\%2Bfunzioni\%27\%2Bnell\%27intreccio\%2Bmul tilivello\%2Bdel\%2Bnuovo\%2Btipo\%2Bdi\%2BStato\%2B\%2D\%2Bstato\%2B\%2D\%2Bdottrina\%2B\%2D\%2B.

MORELLI, A. (2017): "Ascese e declini del regionalismo italiano. Quali prospettive dopo i referendum di Lombardia e Veneto?", in Le Regioni, n. 3, p. 321 e ss. URL: https://www.rivisteweb.it/doi/10.1443/88413. DOI: https://doi. org/10.1443/88413.

PAREJO ALFONSO, L. (2019): "Las reformas territoriales en España", in FONT I LLOVET, T.; GALÁN GALÁN, A.; TUBERTINI, C. (a cura di): Las reformas del Estado. Coloquio internacional en homenaje al prof. Luciano Vandelli. p. 48. Madrid: lustel.

STERPA, A. (2019): "La differenziazione possibile: istituire le macro-Regioni per ridisegnare la mappa delle diversità", in Diritti regionali. Rivista di diritto delle autonomie territoriali, núm. 2. URL: https://www.dirittiregionali.it/wp-content/ uploads/2019/08/documento-integrale-1.pdf.

VANDELLI, L. (2019): "Las relaciones entre el territorio y las autonomías en Europa y América latina", in Jornadas sobre la organización territorial de los Estados: experiencias y problemas en América y Europa, Salamanca, 21 y 22 de enero de 2019. Salamanca: Universidad de Salamanca. Disponible en: $h t t p s: / / w w w . y o u t u b e . c o m / w a t c h ? v=t$ HCPQ5ukLYc\&feature=youtu.be.

28 Su cui si veda la nota sentenza della Corte costituzionale, n. 50 del 2015. 